Revista de Matemática: Teoría y Aplicaciones 2004 11(1) : 81-93

CIMPA - UCR - CCSS ISSN: 1409-2433

\title{
FITTING NON-GAUSSIAN MODELS TO FINANCIAL DATA: AN EMPIRICAL STUDY
}

\author{
Pablo Olivares * Alexánder Alvarez ${ }^{\dagger}$
}

Received/Recibido: 24 Oct 2003

\begin{abstract}
In this paper are presented some experiences about the modeling of financial data by three classes of models as alternative to Gaussian Linear models. Dynamic Volatility, Stable Lévy and Diffusion with Jumps models are considered. The techniques are illustrated with some examples of financial series on currency, futures and indexes.
\end{abstract}

Keywords: Dynamic Volatility, Stable Processes, Diffusions with Jumps, Likelihood.

\section{Resumen}

En el trabajo se presentan algunas experiencias en la modelación de datos financieros usando tres clases de modelos alternativos a los modelos Gaussianos lineales. Se consoderan modelos con volatilidad dinámica, estables de Lévy y difusiones con Saltos. Las técnicas son ilustradas con ejemplos de series financieras de tasas de cambio, futuros e índices.

Palabras clave: Volatilidad Dinámica, Procesos Estables, Difusiones con Saltos, Verosimilitud.

Mathematics Subject Classification: 60H10, 62M10, 62P20, 90A20.

\section{Introduction}

Linear Gaussian models have been considered in the past years to model financial data. In the discrete time context Autoregressive Moving Average processes(ARMA) are considered (see Box-Jenkins (70), [3]). They are defined by:

$$
X_{t}-a_{1} X_{t-1}-\cdots-a_{p} X_{t-p}=e_{t}+b_{1} e_{t-1}+\ldots+b_{q} e_{t-q}
$$

\footnotetext{
*Instituto de Cibernética, Matemática y Física (ICIMAF), La Habana, Cuba. E-Mail: pablo@icmf.inf.cu

${ }^{\dagger}$ Universidad de la Habana, Facultad de Matemática y Computación. San Lazaro y L, Vedado. Ciudad Habana, Cuba. E-Mail: alex@matcom.uh.cu
} 
where $\left(e_{t}\right)_{t \in \mathbb{N}}$ are independent distributed Gaussian r.v. with zero mean and constant variance $\sigma^{2}$. Here $X_{1}, X_{2}, \cdots, X_{n}$ are the spot prices of some financial asset during some periods of time.

In the continuous time framework the following diffusion model is proposed:

$$
d X_{t}=b\left(X_{t}, \theta\right) d t+\sigma\left(X_{t}\right) d B_{t}
$$

where $\theta$ is an unknown parameter, $b\left(X_{t}, \theta\right)$ is the drift, $\sigma\left(X_{t}\right)$ is the variance or the volatility process and $\left(B_{t}\right)_{t \in \mathbb{N}}$ is a standard Brownian Motion. In particular we get the very known Black-Scholes model:

$$
d X_{t}=\mu X_{t} d t+\sigma X_{t} d B_{t}
$$

By Itô formula its solution is given by:

$$
X_{t}=X_{0} e^{\mu t-\frac{1}{2} \sigma^{2} t+\sigma B_{t}}
$$

which implies that marginal distribution laws of spot prices are lognormals.

In the last years empirical evidences against Gaussian Linear models have been accumulated. Despite the diversity of financial series some common stylized facts (see Cont(1999), [5]) not explained by the models above are present, among them we have:

- cluster volatilities

- heavy tails

- returns non autocorrelated

- asymmetry in profits and lost

- "long memory" property

- self-similarity

We will concentrated in the first two aspects. Cluster volatility refers to the fact that periods with high activity in the market altern with others where prices don't present large fluctuations. Moreover, these phenomena seems to take place at random intervals. Heavy tails is linked to the decay to zero of the marginal density of returns at a lesser rate than in the normal distribution, hence, extreme events are observed more frequently.

In order to illustrate these empirical facts in figure 1 prices of sugar futures and the mexican peso vs. dollar exchange rates are shown.

We remark that, according to the graphs, that extreme values are more frequent than expected in presence of normality (see also table 1 ). In adition the volatility might be changing along time. 

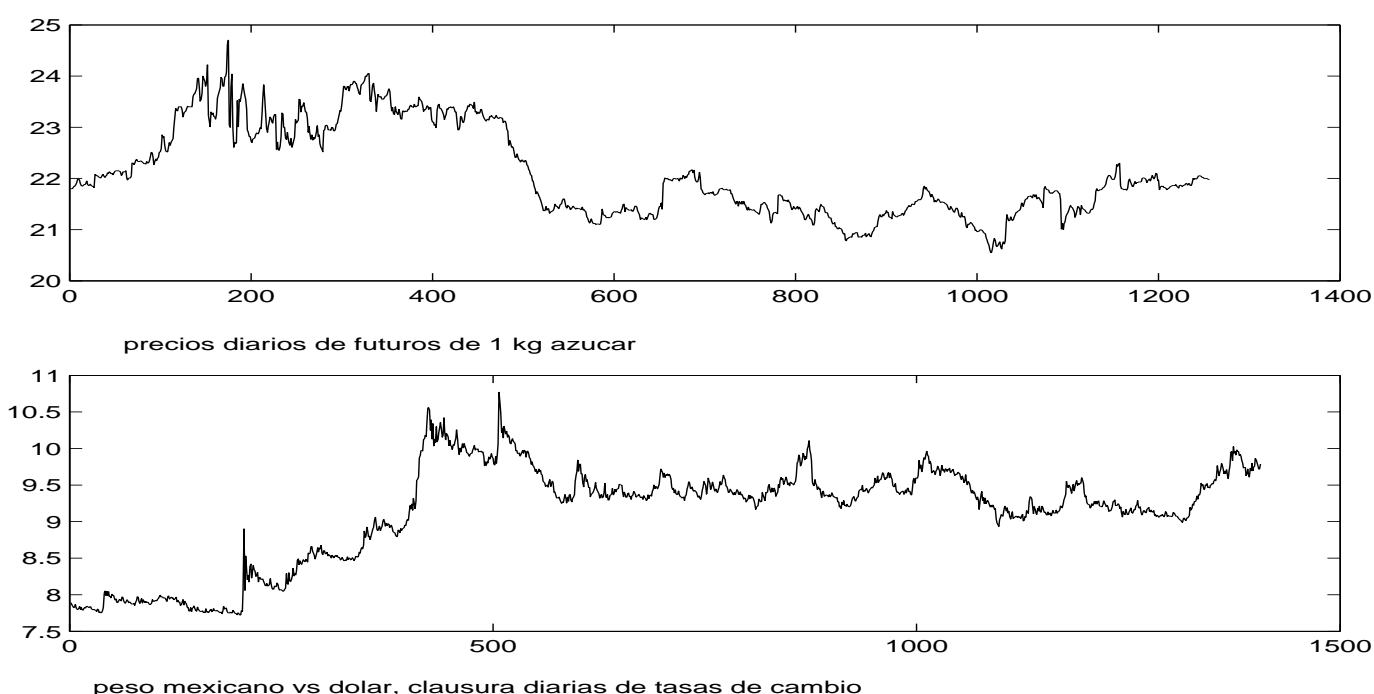

Figure 1: Daily prices of sugar futures(above) and exchange rate of mexican peso vs. US dollar(bellow).
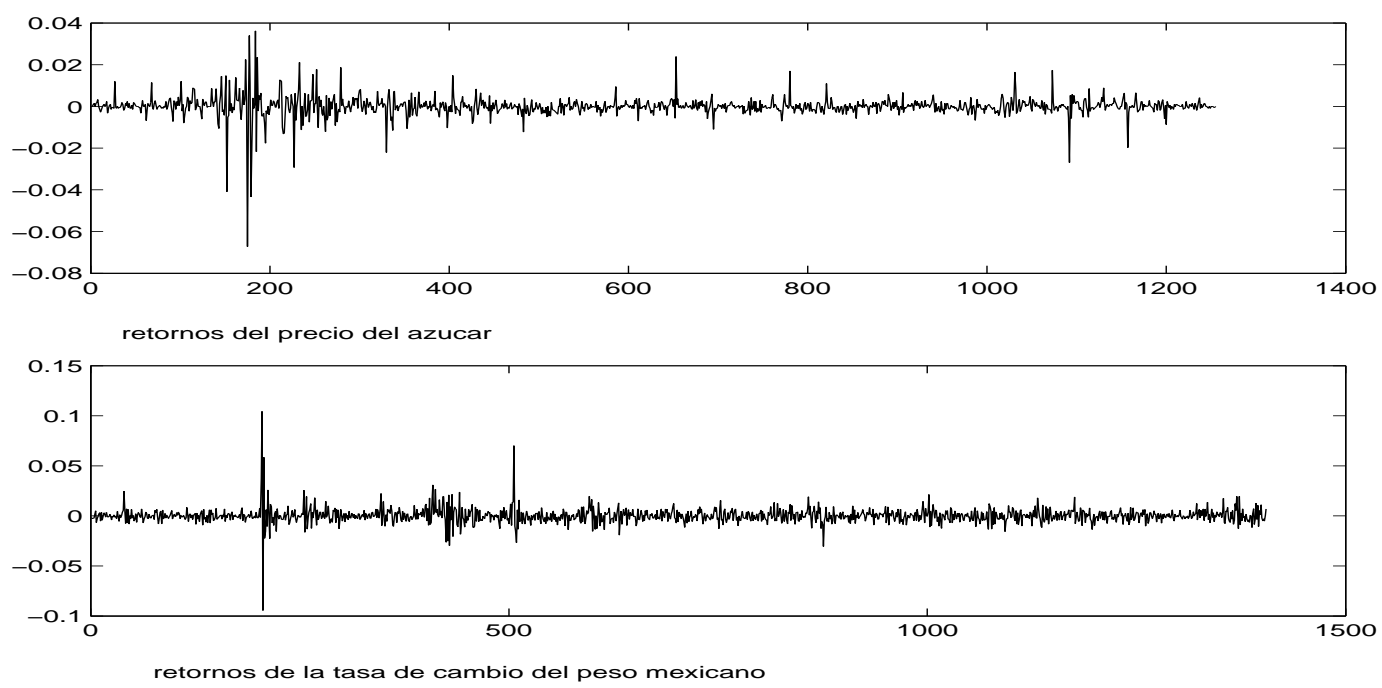

Figure 2: Returns of sugar futures prices(above) and exchange rate of the mexican peso vs. US dollar (bellow). 
Returns are more relevant from the financial point of view, also the returns series is stationary in the mean. They are defined as:

$$
Y_{t}=\frac{X_{t}-X_{t-1}}{X_{t-1}}
$$

or

$$
Y_{t}=\ln \left(\frac{X_{t}}{X_{t-1}}\right)
$$

In figure 2 the returns series of the sugar prices and the mexican peso exchange rate are presented. Also in figures 3 and 4 the logarithms EURO returns and the YEN are shown.

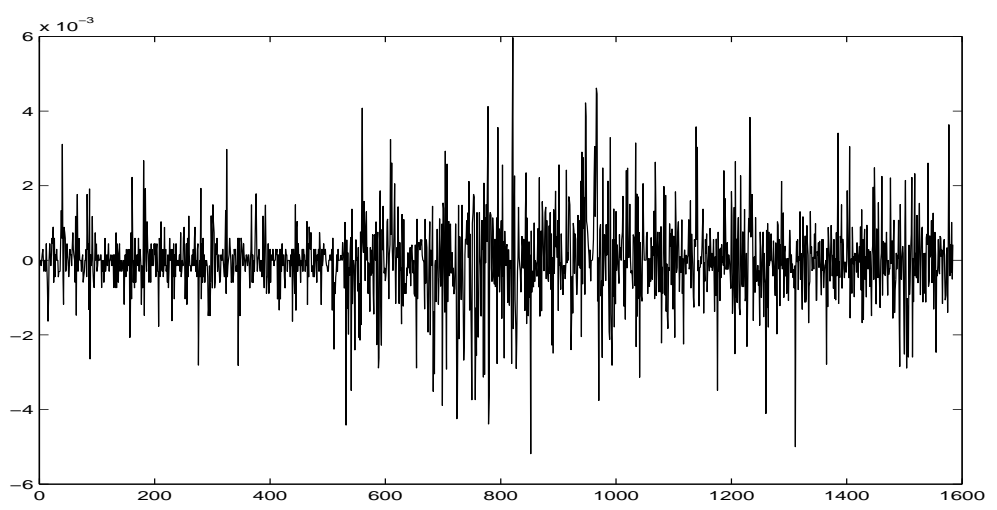

Figure 3: Returns of daily exchange rate EURO/Dollar.

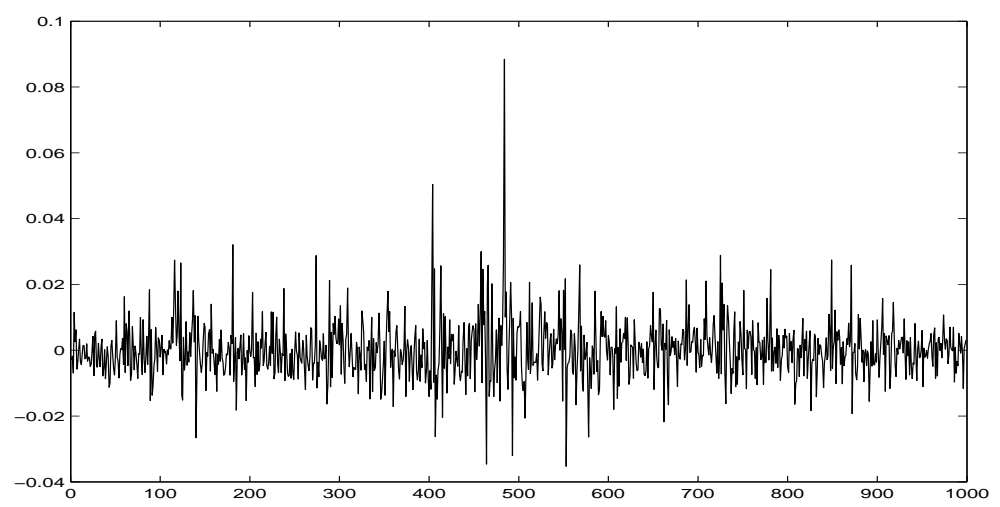

Figure 4: Returns of daily exchange rate YEN/Dollar.

A statistic summary of different return series is shown in table 1.

Note that kurtosis, actually the diffrence with Gaussian kurtosis which equals to 3, is also larger than in the Gaussian case, specially for the sugar future price and the mexican peso. 


\begin{tabular}{c|cccccc}
\hline \hline Asset & Mean & Minimum & Maximum & Std.Dev. & Skewness & Kurtosis \\
\hline Sugar & 0.0000 & -0.0672 & 0.0361 & 0.0048 & -2.5409 & 46.7911 \\
Mexican Peso & 0.0002 & -0.0944 & 0.1042 & 0.0076 & 1.4721 & 49.4355 \\
EURO & -00220 & -.022615 & .023646 & .000033 & .211994 & 5.087970 \\
YEN & .000093 & -.035293 & .088448 & .008802 & 1.413142 & 15.07147 \\
\hline \hline
\end{tabular}

Table 1: Statistic summary of sugar future daily prices, mexican peso, EURO and YEN daily exchange rates versus US dollar.

\section{Dynamic volatility models}

\section{$2.1 \quad \mathrm{ARCH} / \mathrm{GARCH}$ models}

Autoregressive Conditional Heteroscedastic (ARCH) and Generalized Autoregressive Conditional Heterocesdastic models(GARCH) are introduced in the 80's (see Engle(1995), [4] and Bollerslev(1992), [2]). A GARCH model is defined by:

$$
\begin{aligned}
Y_{t} & =c_{0}+\sum_{i=1}^{r} c_{i} Y_{t-i}+\sigma_{t} \varepsilon_{t} \\
\sigma_{t}^{2} & =k+\sum_{i=1}^{p} a_{i} Y_{t-i}^{2}+\sum_{i=1}^{q} b_{i} \sigma_{t-i}^{2}
\end{aligned}
$$

where $\left(\varepsilon_{t}\right)$ are independent random variables with standard normal distribution.

Remark 2.1. 1. For simplicity we consider $c_{i}=0$ for $i=0,1,2$, cdots, $r$

2. $X_{t}$ are, conditionally to $\sigma_{t}$, Gaussian distributed. Its common marginal density is a mixing of Gaussian laws, which has heavy tails.

3. The model also captures the persistence of the volatility. Indeed, according to equation (7), large values of the return process at present time correspond with large values of the next observation of the series, which in turn implies large values of the volatility and so on.

The statistical analysis is usually divided in three parts:

- A preliminary analysis for identification purpose is carry on trough the autocorrelation function(ACF) and the partial autocorrelation function(PACF). Also a LjungBox-Pierce Q-test for a departure from randomness based on the ACF of the data is implemented.

- A parameter estimation is performed based on the likelihood criteria, using numerical techniques to obtain the maximum values. We follow a sequential quadratic optimization method as numerical procedure.

- A diagnosis test of the model via analysis of residuals is performed. 
The techniques are illustrated with the analysis of the EURO series mentioned above. In figure 5 autocorrelations of the series are shown. Note that in the case of the EURO all values are significative equal to zero, except lags 4 and lags 18 which are slightly outside the confidence interval but very close to the confidence band. We consider they are not significative also.

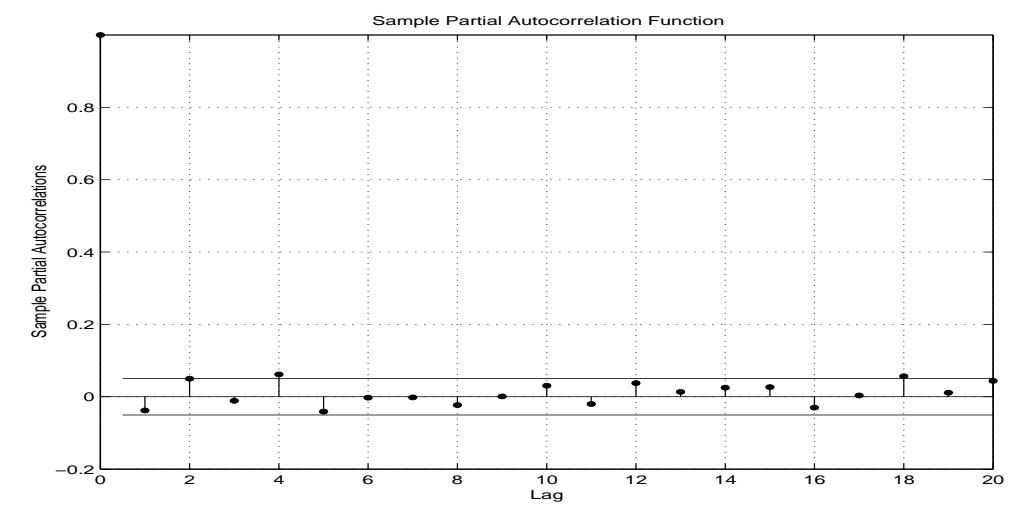

Figure 5: Autocorrelacion function and partial autocorrelation function for daily EURO returns series.

In the case of the sugar future prices and the mexican peso other non zero correlations appear. A further ARMA ajustment is needed.

On the other hand figure 6 shows the values of the ACF for the squares of the EURO return series. They reveal the existence of autocorrelations, hence data exhibits non-linear dependence indicating a possible non Gaussian distribution.

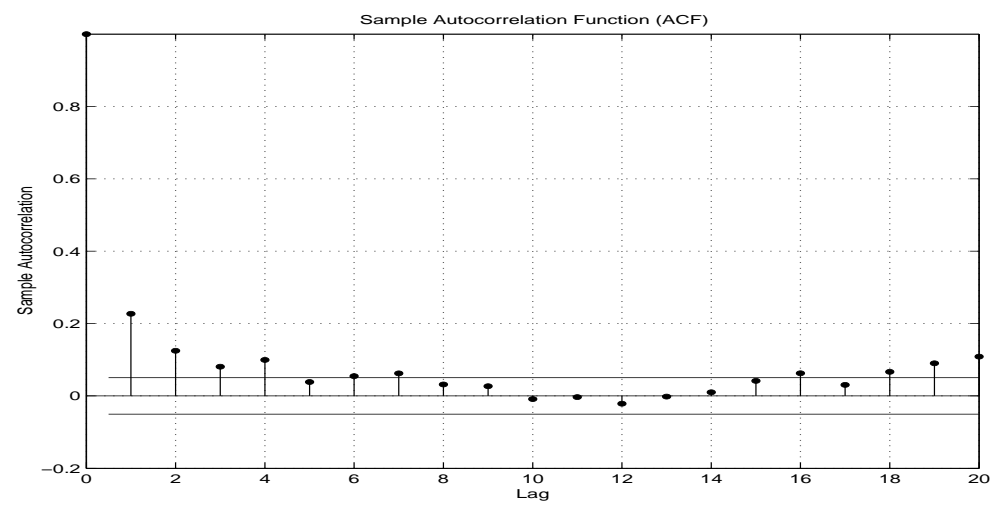

Figure 6: Autocorrelacion function and partial autocorrelation function for squares daily EURO returns series.

Also a test for randomness is performed. Results of the Ljung-Box-Pierce Q-test for 


\begin{tabular}{c|ccc}
\hline \hline Currency & $p$-value & L-B-P Statistic & Critical value at 5\% \\
\hline EURO & 0.03674156557929 & 32.66132331404926 & 31.41043284423092 \\
\hline \hline
\end{tabular}

Table 2: Ljung-Box-Pierce Q-test for the EURO returns.

\begin{tabular}{c|ccc}
\hline \hline Parameter & Statistic Value & Standard Error & $T$ \\
\hline$c_{0}$ & $-2.1877 \times 10^{-5}$ & $2.2427 e-005$ & -0.9755 \\
$k$ & $2.3674 \times 10^{-7}$ & $2.424 \times 10^{-8}$ & 9.7663 \\
$b_{1}$ & 0.54041 & 0.036768 & 14.6978 \\
$a_{1}$ & 0.29113 & 0.032936 & 8.8394 \\
\hline \hline
\end{tabular}

Table 3: Fit of a GARCH(1,1): estimation results for the Euro return series.

the EURO returns are shown in table 2. Th null hypotheses of randomness is rejected at $5 \%$ significance level.

The conditional likelihood of the data is:

$$
\begin{aligned}
l\left(y, k, a_{i}, b_{i} / \sigma_{t}\right) & =\sum_{t=1}^{n} \log f\left(y_{t}, k, a_{i}, b_{i} / \sigma_{t}\right) \\
& =\sum_{t=1}^{n} \log \frac{1}{\sigma_{t}}+\sum_{t=1}^{n} \log f_{\varepsilon_{t}}\left(y_{t}\right)
\end{aligned}
$$

where $f_{\varepsilon_{t}}\left(x_{t}\right)$ is the marginal density of the normal noise $\varepsilon_{t}$. We illustrate the techniques by fitting a $\operatorname{GARCH}(1,1)$. The maximization procedure is done using MATLAB optimization subroutine GARCHFIT.

Note that both intercepts stay in the confidence interval of zero. Also the sum $a_{1}+$ $b_{1}<1$ indicating a stationary behavior (Shiriaev(1999), [12]). Once the estimation of parameters is done a residual analysis is implemented. In figure 7 the adjusted variance is shown as well as the adjusted residuals according to equation (7). Again, a LjungBox-Pierce Q-test for a departure from randomness of the standardized residuals $\frac{\hat{Y}_{t}}{\hat{\sigma}_{t}}$ is implemented, results are shown in table 4 indicating the non rejection of the random white noise in this occasion.

\begin{tabular}{|c|c|c|}
\hline \hline$p$-value & L-B-P Statistic & Critical value \\
\hline 0.69804810136988 & 7.28755024134759 & 18.30703805327515 \\
0.84651541062226 & 9.55917544804852 & 24.99579013972862 \\
0.70549399299311 & 16.17844373146159 & 31.41043284423092 \\
\hline \hline
\end{tabular}

Table 4: Ljung-Box-Pierce Q-test for a departure from randomness of the standardized residuals. 

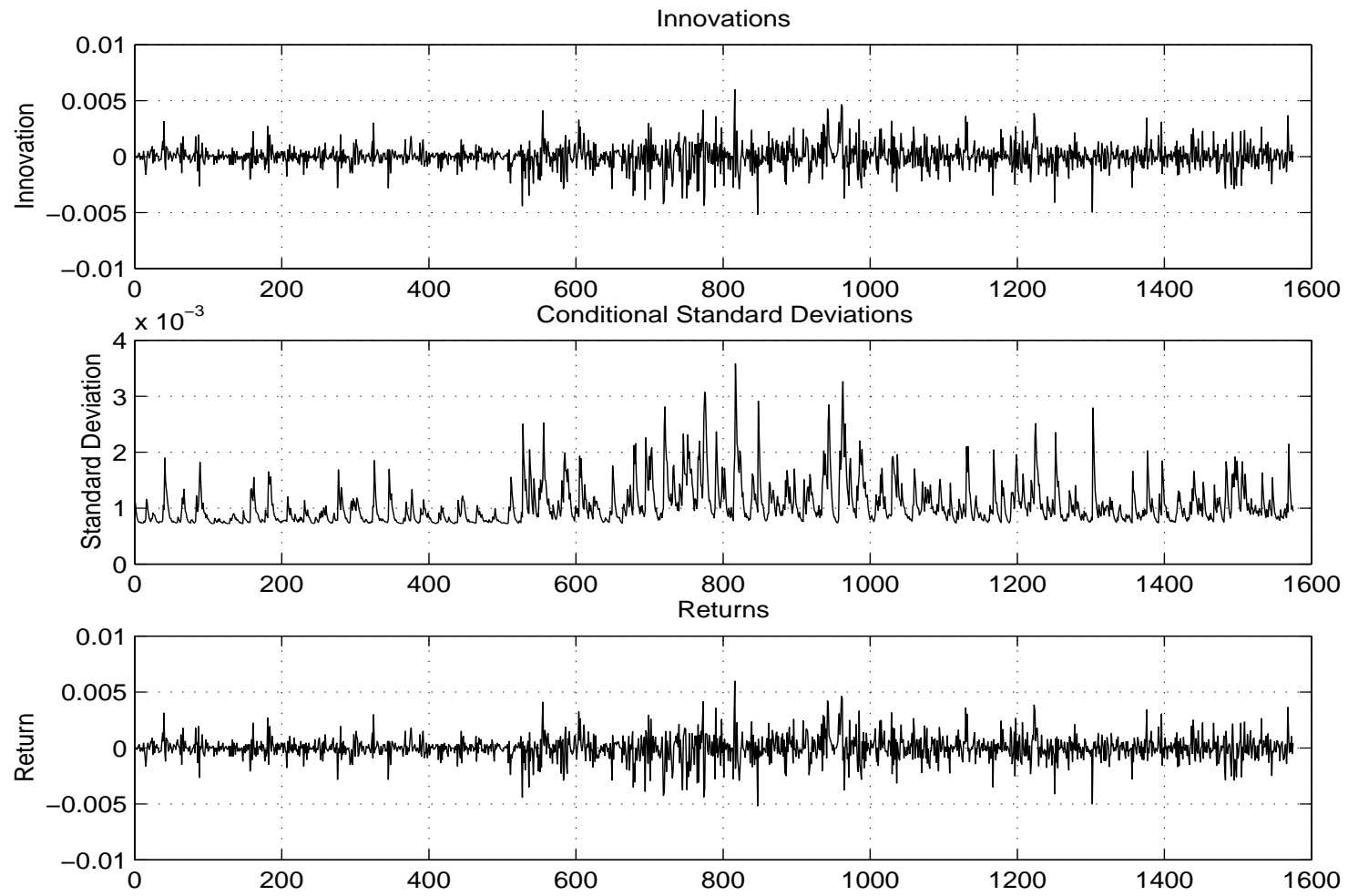

Figure 7: Innovations, estimated variance and returns for daily EURO returns series.

\section{$3 \quad$ Stable noises and stable Lévy processes}

In order to explain heavy tails of the marginal distribution of the returns several distribution laws have been proposed. The stable distribution is one of them, it is introduced in (Fama(1971),[6]) and (Mandelbrot(1963),[8]). The following conditions are equivalent definitions of a stable random variable $X$ :

a) $X$ has a domain of attraction, i.e., there are a sequence of i.i.d. random variables $\left\{Y_{i}\right\}_{i \in \mathbf{N}}$, a real positive sequence $\left\{a_{i}\right\}_{i \in \mathbf{N}}$ and a real sequence $\left\{b_{i}\right\}_{i \in \mathbf{N}}$ such that

$$
\frac{1}{a_{n}} \sum_{i=1}^{n} Y_{i}-b_{n} \rightarrow X \text { in distribution }
$$

b) The characteristic function $(\mathrm{CF})$ of $X$ admits the following form:

$$
\phi_{X} t= \begin{cases}\exp \left(-\sigma^{\alpha}|t|^{\alpha}(1-i \beta \operatorname{sgn}(t) \tan \pi \alpha 2)+i \mu t\right) & \text { for } \alpha \neq 1 \\ \exp \left(-\sigma|t|\left(1+i \beta \frac{\pi}{2} \operatorname{sgn}(t) \log |t|\right)+i \mu t\right) & \text { for } \alpha=1\end{cases}
$$




\begin{tabular}{c|cccc}
\hline \hline Currency & Tail Index & Symmetry Index & Scaling Index & Location Index \\
\hline Can Dollar & 1.7660 & 0.0100 & 0.0021 & -0.0001 \\
British Pound & 1.6490 & 0.1600 & 0.0029 & -0.0000 \\
Yen & 1.6490 & 0.2200 & 0.0047 & 0.0003 \\
EURO & 1.5710 & -0.5600 & 0.0205 & -0.0023 \\
\hline \hline
\end{tabular}

Table 5: McCulloch estimates for daily currency data.

where the parameters satisfy the constraints $\alpha \in(0,2], \sigma \in \mathbf{R}_{0}^{+}, \beta \in[-1,1]$ and $\mu \in$ R.

A random variable $X$ with stable distribution of parameters $\alpha, \beta, \sigma, \mu$ is denoted by $X \sim S(\alpha, \beta, \sigma, \mu)$. It is well known (see for example (Taqqu and Samorodnitsky (1994),[10]) that for $\alpha \in(0,2)$ and $X \sim S(\alpha, \beta, \sigma, 0)$ we have

$$
\lim _{x \rightarrow \infty} x^{\alpha} P(X>x)=C_{\alpha}(1+\beta) \sigma^{\alpha}
$$

Hence $\alpha$ is an index about the thickness of the tail. Also $\beta$ is a coefficient for symmetry (note that for $\beta=0$ the $\mathrm{CF}$ is real then the density law is symmetric), $\sigma$ is a scale parameter and $\mu$ is a location parameter.

A close expression for the density is in general unknown with the exception of $\alpha=$ $2 \operatorname{textand} \beta=0$ where the Gaussian law is obtained and $\alpha=1$ and $\beta=0$ where the Cauchy law is obtained.

Another important property is that $E|X|^{p}$ exists for $0<p<\alpha$ and $E|X|^{p}=\infty$ for $p \geq \alpha$. For example variance is only finite in the Gaussian case.

Several estimation procedures have been implemented for stable laws, for a review, discussions and comparisons see Seco et al.(2003), [11] or Weron(2000), [14]. Four kind of estimators can be distinguished:

- tail estimators (only for $\alpha$ ),

- likelihood estimators,

- quantiles and moments estimators,

- sample characteristic estimators.

A simulation study, (Seco et al.(2003), [11]) reveals that the McCulloch quantile based estimator seems to be a better compromise between accuracy and speed, see also (McCulloch(1979), [9]). Table 5 shows estimates obtained for exchange rates of four currencies using McCulloch method. Note that all of them exhibit a tail behavior heavier than normal, with the EURO return series showing a negative asymmetry.

A similar result is found for NASDAQ and Dow Jones indexes. Additionally we performed a Kolmogorov-Smirnov test showing that the stable hypothesis is not rejected. Results can be seen in table 6 . 


\begin{tabular}{c|ccc|c}
\hline \hline Currency & K-S Statistics & $\begin{array}{c}\text { Critical Value } \\
\text { at } 1 \%\end{array}$ & $\begin{array}{c}\text { Critical Value } \\
\text { at } 5 \%\end{array}$ & Conclusion \\
\hline Can Dollar & 0.6637 & 1.63 & 1.36 & No Rejected \\
British Pound & 0.6034 & 1.63 & 1.36 & No Rejected \\
Yen & 0.8925 & 1.63 & 1.36 & No Rejected \\
EURO & 1.0942 & 1.63 & 1.36 & No Rejected \\
\hline \hline
\end{tabular}

Table 6: Kolmogorov-Smirnov test for stable distribution.

Also self-similarity property of stable distribution is tested with the change of scale from weekly to daily data for NASDAQ and Dow Jones indexes. Indeed, if we consider an stable Levy process, i.e., a process $\left(X_{t}\right)_{t \geq 0}$ with independent increments and $X_{t} \sim S(\alpha, \beta, \sigma, \mu)$. From here it is easy to see that $X_{c t}$ and $c^{\frac{1}{\alpha}} X_{t}+\mu\left(c-c^{\frac{1}{\alpha}}\right)$ have the same distribution law. Here $c>0$ is the new time scale considered. Therefore a two side Kolmogorov-Smirnov test is implemented to test

$$
H_{0}: X_{c t}=c^{\frac{1}{\alpha}} X_{t}+\mu\left(c-c^{\frac{1}{\alpha}}\right) \text { in distribution }
$$

The null hypotheses of self-similarity is not rejected for this particular scale, the results can be seen in table 7 .

\begin{tabular}{c|ccc}
\hline \hline Index & Two Side K-S statistic & $p$-value & decision \\
\hline Dow Jones & .6655 & .7759 & non-rejected \\
NASDAQ & 1.3291 & .0566 & non-rejected \\
\hline \hline
\end{tabular}

Table 7: Self-similarity test for Dow Jones and NASDAQ.

\section{Diffusion with jumps models}

Another attempt to explain large fluctuations in the return prices is to consider, together with normal noises, an extra term in equation (2) related to the jumps. Usually a pure jump Markov process is considered, in particular we have:

$$
d X_{t}=b\left(X_{t^{-}}, \theta\right) d t+\sigma\left(X_{t^{-}}\right) d B_{t}+\gamma\left(X_{t^{-}}, \theta\right) d Z_{t}
$$

where $\left(Z_{t}\right)_{t \in \mathbb{N}}$ is a compound Poisson process given by

$$
Z_{t}=\sum_{i=1}^{N_{t}} X_{i}
$$

$X_{1}, X_{2}, \cdots, X_{n}$ are independent identical distributed random variables with density $g(\theta,$. of mean $\mu$ and $\left(N_{t}\right)_{t \in \mathbb{N}}$ is a Poisson Process of intensity $\lambda(\theta)$. Here $\theta$ is the unkown parameter. The loglikelihood, for continuous observations on $[0, T]$, is obtained from 
generalized Girsanov theorem (see Jacod and Shiriaev (1987), [7]). Disregarding the terms non depending on $\theta$, it is given by:

$$
\begin{aligned}
l_{t}(\theta)= & \int_{0}^{t} b\left(\theta, X_{s^{-}}\right)^{\prime} \sigma_{s}^{-1} d X_{s}^{c}-\frac{1}{2} \int_{0}^{t} b\left(\theta, X_{s^{-}}\right)^{\prime} \sigma_{s}^{-1} b\left(\theta, X_{s^{-}}\right) d s \\
& +N_{t} \log [\lambda(\theta)]-t \lambda(\theta) \\
& +\sum_{s \in S_{t}} \log \left[g\left(\theta, \varphi\left(\theta, X_{s-}, \Delta_{s}\right)\right)\left|J_{\varphi}\left(\theta, X_{s-}, \Delta X_{s}\right)\right|\right]
\end{aligned}
$$

Here $S_{t}=\left\{s \leq t: \Delta X_{s} \neq 0\right\}$ is the set of jumps times on $[0, T]$.

Also :

$$
X_{t}^{c}(\theta)=X_{t}-x_{0}-\sum_{s \leq t} \Delta X_{s}-\int_{0}^{t} b\left(\theta, X_{s}\right) d s
$$

is the continuous part of the process, $\varphi$ is the inverse function of $\gamma$ and $\left|J_{\varphi}\right|$ the deteminant of its Jacobian matrix.

Asymptotic behavior results for the m.l.e. under continuous observations are obtained in (Sorensen(1990),[13]).

For discrete observations of equal length $\Delta$ the likelihood function only can be calculated in an approximate way via the discretization of (15). For an exponential family (see a precise concept in Sorensen(1990),[13])and a parametric space a subset of $\mathbb{R}^{3}$, with parameters $(\theta, \lambda, \mu)$ corresponding to the drift, the intensity of the Poisson Process and the mean of the jumps respectively it is obtained that:

$$
\begin{aligned}
\widetilde{l}_{n}^{n, T, h^{\prime}}(\theta, \lambda, \mu)= & \sum_{i=1}^{n} b_{\Delta(i-1)} \sigma_{\Delta(i-1)}^{-1}\left(\widetilde{X}^{c}\left(n, h^{\prime}\right)_{\Delta i}-\widetilde{X}^{c}\left(n, h^{\prime}\right)_{\Delta(i-1)}\right)- \\
& -\frac{\Delta}{2} \sum_{i=1}^{n} b_{\Delta(i-1)} \sigma_{\Delta(i-1)}^{-1} b_{\Delta(i-1)}(\theta)+ \\
& +\widetilde{N}\left(n, h^{\prime}\right)_{t} \log \lambda-t \lambda+ \\
& +\sum_{\Delta i \in \widetilde{S}\left(n, h^{\prime}\right)_{t}} \log \left[g\left(\mu, \varphi\left(\mu, \Delta i, X_{\Delta i}, \widetilde{\Delta X}\left(n, h^{\prime}\right)_{\Delta i}\right)\right)\right] \times \\
& \times\left|J_{\varphi}\left(\mu, \Delta i, X_{\Delta i} \widetilde{\Delta X}\left(n, h^{\prime}\right)_{\Delta i}\right)\right|
\end{aligned}
$$

where

$$
\widetilde{X}^{c}\left(n, h^{\prime}\right)_{\Delta(i+1)}= \begin{cases}\widetilde{X}^{c}\left(n, h^{\prime}\right)_{\Delta i}+X_{\Delta(i+1)}-X_{\Delta i} & \text { si }\left\|X_{\Delta(i+1)}-X_{\Delta i}\right\|<h^{\prime} \\ \widetilde{X}^{c}\left(n, h^{\prime}\right)_{\Delta i} & \text { si }\left\|X_{\Delta(i+1)}-X_{\Delta i}\right\|>h^{\prime}\end{cases}
$$

for a given fixed number $h^{\prime}$ is an approximation of the continuous part,

$$
\widetilde{N}\left(n, h^{\prime}\right)_{t}=\sum_{\Delta i<t} 1_{\left[\left\|X_{\Delta(i+1)}-X_{\Delta i}\right\|>h^{\prime}\right]}
$$




\begin{tabular}{l|lll}
\hline \hline & $T=20$ & $T=40$ & $T=100$ \\
\hline $\bar{\theta}$ & -1.422 & -1.202 & -1.085 \\
$\bar{\alpha}$ & 18.54 & 14.11 & 11.71 \\
$\bar{\lambda}$ & 0.541 & 0.527 & 0.498 \\
$\bar{\mu}$ & 4.947 & 4.962 & 4.981 \\
\hline \hline
\end{tabular}

Table 8: Estimators from simulated mean-reverting process with Poisson jumps with $\theta=-1, \alpha=10, \sigma=1, \lambda=0.5, \mu=5, h=0.001, \Delta=0.001, h^{\prime}=0.5, n=10$.

is an approximation of the number of jumps,

$$
\widetilde{S}\left(n, h^{\prime}\right)_{t}=\left\{\Delta i:\left\|X_{\Delta(i+1)}-X_{\Delta i}\right\|>h^{\prime}\right\}
$$

is an approximation of $S_{t}$ and $\widetilde{\Delta X}\left(n, h^{\prime}\right)_{t}=1_{\left[t=\Delta i \wedge\left\|X_{\Delta(i+1)}-X_{\Delta i}\right\|>h^{\prime}\right]}\left(X_{\Delta(i+1)}-X_{\Delta i}\right)$ as approximation of $\Delta X_{t}$.

Solving

$$
\dot{\vec{l}}_{n}^{n, T, h^{\prime}}(\theta, \lambda, \mu)=0
$$

we get the discretized m.l.e $\widehat{\theta}_{T}^{n, h^{\prime}}$.

In (Alvarez et al.(2003), [1]), the asymptotic behavior of the m.l.e. estimators is studied and numerical results are illustrated from simulation data. For a mean-reverting with jumps stochastic equation:

$$
d X_{t}=\left(\alpha+\theta X_{t}\right) d t+\sigma d B_{t}+d Z_{t}
$$

where $\left(Z_{t}\right)_{t \geq 0}$ is again a compond Poisson Process with parameters $\lambda$ and $\mu$ results can be seen in table 8 .

\section{Conclusions and recommendations}

The first two alternatives capture the heavy tail behavior of financial data for the examples considered. The diffusion with jumps model needs to be confronted with real data, nevertheless it perform well in preliminary simulation studies. Also a comparison between the different models could be useful.

The models presented are not, by far, the sole alternatives to the Gaussian Linear models in order to explain the stilyzed facts of financial data, for example stochastic volatility and switching models could also be considered.

\section{References}

[1] Alvarez, A.; Olivares, P. (2003) "M.l.e. for diffusion with jumps from discrete observations", Reporte de Investigación. ICIMAF, 253-2003. 
[2] Bollerslev, T.; Chou, R.C.; Kroner, K. (1992) "ARCH modeling in finance", Journal of Econometrics 52: 5-59.

[3] Box, G.E.; Jenkins, G.M. (1976) Time Series Analysis, Forecasting and Control. Holden-Day.

[4] Engle, R.F. (1995) ARCH: Selected Readings. Oxford University Press, Oxford.

[5] Cont, R. (1999) "Statistical properties of financial time series". Symposium on Mathematical Finance Fudan University, Shanghai.

[6] Fama, E.; Roll, R. (1971) "Parameters estimates for symmetric stable distributions", Journal of the American Statistical Association 66: 331-339.

[7] Jacod, J.; Shiryaev, A.N. (1987) Limit Theorems for Stochastic Processes. Springer Verlag, New York.

[8] Mandelbrot, B. (1963) "The variation of certain speculative prices", J. of Bus. 26: $394-419$.

[9] McCulloch, J.H. (1963) "Measuring tail thickness in order to estimate the stable index $\alpha$ : a critique", Bussiness and Economic Statistics 15: 74-81.

[10] Samorodnitsky, G.; Taqqu, M.S. (1994) Stable non-Gaussian Random Processes: Stochastic Models with Infinite Variance. Chapman \& Hall, London.

[11] Seco, L.; Alvarez, A.; Escobar, M.; Olivares, P. (2003) "An overview of estimation methods for stable distributions with financial applications", Working Paper.

[12] Shiriev, A.N. (1999) Essencials of Stochastic Finance. World Scientific, Singapur.

[13] Sørensen, M. (1990) "Likelihood methods for diffusions with jumps", Statistical Inference in Stochastic Processes. N.U. Prabhu \& I.V. Basawa (Eds.). Marcel Dekker, New York: 67-106.

[14] Weron R. (s.f.) "Performance of the etimators of stable laws", Working paper. 